\title{
Prevalence and incidence of chronic wounds and related complications: a protocol for a systematic review
}

Krister Järbrink1, Gao Ni ${ }^{1}$, Henrik Sönnergren², Artur Schmidtchen ${ }^{3}$, Caroline Pang ${ }^{4}$, Ram Bajpai ${ }^{1}$ and Josip Car $^{1,5^{*}}$ (D)

\begin{abstract}
Background: Chronic wounds impose a significant and often underappreciated burden to the individual, the healthcare system and the society as a whole. Preliminary literature search suggests that there are at present no reliable estimates on the total prevalence of chronic wounds for different settings and categories of chronic wounds. Such information is essential for policy and planning purposes as the increasing number of elderly and the prevalence of lifestyle diseases point in the direction of an increased burden. Knowledge about the prevalence and incidence of chronic wounds in relation to population characteristics is important for informing healthcare planning and resource allocation. The objective is to present a transparent process for how to review the existing literature on the prevalence and incidence rates of chronic wounds and resulting implications.

Methods/design: We will search electronic bibliographic databases (MEDLINE, EMBASE, the EBM Reviews and Cochrane, Cumulative Index to Nursing and allied Health Literature (CINAHL), PsycINFO, Global Health) and reference lists of included articles. Two investigators will independently screen titles and abstracts and select studies involving adults with chronic wounds. These investigators will also independently extract data using a pre-designed data extraction form that will cover information on demographics, diagnostics including disease prevalence, medical history, hospital and community-based management and outcomes. Subgroup analysis and sensitivity analysis will be performed to address the heterogeneity across studies. Meta-analysis will also be performed if homogeneous group of studies will be found. The collective evidence will be further stratified according to the important background variables if allowed.
\end{abstract}

Discussion: This study will describe the available epidemiological evidence and summarise prevalence and incidence rates of chronic wounds and related complications. A better understanding of the relationship between population profile and the prevalence of chronic wounds and related complications will be helpful in the development of guidelines for patient management.

\section{Systematic review registration: PROSPERO CRD42016037355}

Keywords: Chronic wounds, Hard-to-heal ulcers, Wound infection, Ulcer, Wound healing, Diabetic foot, Amputation, Epidemiology, Prevalence, Incidence

Abbreviations: CINAHL, Cumulative Index to Nursing and allied Health Literature; GRADE, Grading of Recommendations Assessment, Development and Evaluation; PRISMA-P, Preferred Reporting Items for Systematic Reviews and Meta-Analyses Protocols; PROSPERO, International Prospective Register of Systematic Reviews

\footnotetext{
*Correspondence: josip.car@imperial.ac.uk; josip.car@ntu.edu.sg

'Centre for Population Health Sciences (CePHaS), Lee Kong Chian School of

Medicine, Nanyang Technological University, 59 Nanyang Drive, Experimental

Medicine Building, Singapore 636921, Singapore

${ }^{5}$ Global eHealth Unit, Department of Primary Care and Public Health, School

of Public Health, Imperial College London, 3rd Floor Reynolds Building, St

Dunstan's Road, London W6 8RP, UK

Full list of author information is available at the end of the article
} 


\section{Background}

A chronic wound can be defined as one that has failed to proceed through an orderly and timely reparative process to produce anatomic and functional integrity within a period of 3 months or that has proceeded through the repair process without establishing a sustained, anatomic and functional result $[1,2]$. The nomenclature is far from agreed upon, and these wounds are sometimes referred to as hard-to-heal or difficult-to-heal wounds/ulcers, and the time span required for chronicity has been defined in the range 4 weeks up to more than 3 months [2-4]. Based on the causative aetiologies, the Wound Healing Society classifies chronic wounds into four categories: pressure ulcers, diabetic ulcers, venous ulcers and arterial insufficiency ulcers [5]. Chronic wounds are often termed ulcers and can be defined as wounds with a full thickness in depth and a slow healing tendency. Often disguised as a comorbid condition, chronic wounds represent a silent epidemic that affects a large fraction of the world population [6]. It is estimated that 1 to $2 \%$ of the population will experience a chronic wound during their lifetime in developed countries [7]. The dramatic increase in the ageing population will increase these numbers as wound closure is negatively associated with age [8]. Complications of chronic wounds include infection such as cellulitis and infective venous eczema, gangrene, haemorrhage and lower-extremity amputations. Chronic wounds lead to disability and disability worsens wound outcomes resulting in a vicious cycle [9].

Due to the low base rate of complete healing in the natural history [10], chronic wounds have a significant impact on the health and quality of life of patients and their families, causing pain, loss of function and mobility, depression, distress and anxiety, embarrassment and social isolation, financial burden, prolonged hospital stays and chronic morbidity or even death [11]. Evidence suggests that chronic wounds impose significant and often underappreciated burden to the individual, the healthcare system and the society as a whole $[12,13]$. In the USA, for example, chronic wounds are reported to affect 6.5 million patients with more than US $\$ 25$ billion each year spent by the healthcare system on treating wound-related complications [6]. The costly nature of chronic wound management is further confirmed with estimates from the UK, where the cost to the National Health Service of caring for patients with chronic wounds were conservatively estimated at US\$3.4-4.6 billion per year (in 2005) representing around $3 \%$ of the total estimated out-turn expenditure on health for the same period [14].

Preliminary literature search suggests that there are at present no reliable estimates on the total prevalence and incidence of chronic wounds for different settings and categories of chronic wounds [15]. The wide disparity of the epidemiological estimates arises mainly from significant heterogeneity in terms of study design and data collection method [15]. The varieties also come from the way of reporting prevalence: some report point prevalence while others report period prevalence [15]. The same disparity is also found in incidence reports. We aim to embrace a larger number of databases and a considerable broader search strategy compared to a previous review [15]. We also intend to thoroughly assess and consider the quality of included studies and carefully pay regard to the applied definitions of chronic wounds. These approaches are believed to provide more reliable estimates on the prevalence for different settings and categories of chronic wounds than previously presented. Such information is essential for policy and planning purposes particularly as the increasing number of elderly and the increase in the prevalence of lifestyle diseases further raise the risk for chronic wounds. The literature review is intended to gather the existing knowledge and identify factors of importance to the prevalence of chronic wounds and to the incidence of complications. The focus is on chronic wounds in the categories of pressure ulcers, diabetic ulcers, venous ulcers and arterial insufficiency ulcers.

Specific review questions are:

- What is the prevalence and incidence of chronic wounds in the categories of pressure ulcers, diabetic ulcers, venous ulcers and arterial insufficiency ulcers, for different settings and subgroups according to internationally published studies?

- What is the prevalence and incidence of infections, gangrene, haemorrhage and limb amputation among patients with chronic wounds in the categories of pressure ulcers, diabetic ulcers, venous ulcers and arterial insufficiency ulcers, for different settings and subgroups according to internationally published studies?

Further knowledge about the distribution of chronic wounds and related complications in different settings and among subgroups is essential for informing healthcare planning and resource allocation. Pooling of such data is also necessary to monitor trends in disease burden and to contribute to the design of further etiological studies.

\section{Objectives}

The overall aim of this systematic review protocol is to present a transparent process for how the information will be collected on the prevalence and incidence of chronic wounds and related complications. This will include the key research questions that this systematic review will address, a description of systematic literature 
search strategies, criteria for inclusion or exclusion of studies, a description of coding procedures, study quality measures and statistical procedures for the quantitative analysis of data from eligible studies.

\section{Methods}

Protocol

The methods for this systematic review have been developed according to the recommendations from the Preferred Reporting Items for Systematic Review and Meta-Analysis Protocols (PRISMA-P) 2015 statement [16]. This systematic review protocol has been registered in the International Prospective Register of Systematic reviews (PROSPERO): CRD42016037355). A PRISMA-P file is attached (see Additional file 1).

\section{Eligibility criteria Population}

The population of interest will include adult patients 18 years of age and older with pressure ulcers, diabetic ulcers, venous ulcers and arterial ulcers. Patients with chronic wounds resulting from surgical wounds and skin tumours will be excluded.

\section{Outcome}

The primary outcome will be the point prevalence, period prevalence, cumulative incidence and incidence rate of chronic wounds in the categories of pressure ulcers, diabetic ulcers, venous ulcers and arterial ulcers, and complications thereof in four categories (infections, gangrene, haemorrhage and limb amputation). Secondary outcomes will be dropping out, the reasons for that, and the number of deaths by cause.

\section{Study design}

Studies will be restricted by design and observational studies, cross-sectional studies, cohort studies, casecontrol studies, single arm studies and systemic review/ meta-analyses will be included. Non-research letters and editorials, seminar reviews, case studies, case series and animal studies will be excluded. Randomised controlled trials will be excluded based on methodological inappropriateness of research design for the type of questions to be answered.

\section{Report characteristics}

Searches will be limited to peer-reviewed full text articles in English language, and letters, abstracts and editorials are to be excluded. There will be no geographical limitation on the included studies.

\section{Information sources}

A systematic search of MEDLINE (Ovid), EMBASE (Ovid), EBM Reviews and Cochrane (Ovid), Cumulative Index to
Nursing and Allied Health Literature (CINAHL) (EBSCO), PsycINFO (EBSCO) and Global Health (EBSCO) will be undertaken. As we are primarily interested in the contemporary literature on this topic, we will examine publications from January 2000 through December 2015. Contact with authors for further information will be made when necessary. Detailed inclusion and exclusion criteria are presented in Table 1.

\section{Search strategy}

We will develop a comprehensive database containing all published studies addressing the prevalence and incidence of chronic wounds (including hard-to-heal wound/ulcers) and complications thereof in general populations. To construct a comprehensive set of possible search terms, we list indexing terms (for example, subject headings and subheadings, publication types) and text words used to describe concept clusters (single words or phrases that may appear in titles or abstracts, both in full and in various truncations). For instance, we search pressure ulcer with its keyword "pressure ulcer" with all heading and subheadings, and then we search alternative keywords "pressure sore" or "bed ulcer" or "bed sore" appearing in the title or abstract in the form of full or truncations. We sought further terms from clinicians and librarians, and from published strategies from other groups. The search strategy was developed by the research team in collaboration with an experienced medical research librarian at the Lee Kong Chian School of Medicine. Additional comments and suggestions were also received from an experienced librarian at the Karolinska Institutet and incorporated. The search was revised, as necessary, and the final MEDLINE search is presented in Additional file 2. After MEDLINE strategy is finalised, it will be adapted to the syntax and subject headings of other databases.

The reference lists of all included articles published from January 2000 and beyond will also be searched for any additional sources of information. An additional search will be made using the newly introduced term "pressure injury" [17] and relevant articles added.

\section{Study records}

\section{Data management}

We will implement the search strategies and import all references identified to EndNote. The search results from the different electronic databases will be combined in a single EndNote library and we will remove duplicate records of the same reports.

\section{Selection process}

Two reviewers will independently screen titles and abstracts against eligibility criteria to identify potentially included studies. Specifically, titles and abstracts are 
Table 1 Inclusion and exclusion criteria

\begin{tabular}{lll}
\hline & Inclusion criteria & Exclusion criteria \\
\hline Population & $\begin{array}{l}\text { Adult patients ( } \geq 18 \text { years of age) with chronic wounds in the categories of } \\
\text { pressure ulcers, diabetic ulcers, venous ulcers and arterial insufficiency ulcers } \\
\text { and/or complications thereof in terms of infections, gangrene, haemorrhage } \\
\text { and limb amputation. }\end{array}$ & $\begin{array}{l}\text { Patients with chronic wounds as a result } \\
\text { of surgical wounds and skin tumour. }\end{array}$ \\
Study design & $\begin{array}{l}\text { Observational studies, cross-sectional studies, cohort studies, case-control } \\
\text { studies, single arm studies, systematic review/meta-analyses. }\end{array}$ & $\begin{array}{l}\text { Randomised controlled trials, case studies, } \\
\text { case series. }\end{array}$ \\
Outcome & $\begin{array}{l}\text { Point prevalence, period prevalence, cumulative incidence and incidence } \\
\text { rate of chronic wounds in the four categories, and complications thereof. }\end{array}$ & \\
Type of publication & Articles with available full text in English language. & $\begin{array}{l}\text { Abstract, non-research letters and editorials, } \\
\text { seminar reviews. }\end{array}$
\end{tabular}

included if they indicate that it was a population-based or institutional-based study reporting any relevant information on prevalence or incidence of chronic wounds and/or complications thereof. In the next phase, we will retrieve full-text copies of those articles deemed potentially relevant. Two reviewers will independently assess the full text of the retrieved articles for compliance with our eligibility criteria.

Discrepancies between the two reviewers' judgement will be resolved by discussion or by the involvement of a third reviewer. Studies, which appeared to be relevant, but excluded at this stage will be listed in the table "Characteristics of excluded studies", where a reason for exclusion will be noted. Two reviewers will verify the final list of included studies. A PRISMA flow diagram of the study selection procedure will be prepared to provide an overview of the decisions that are made in the data collection process.

\section{Data collection process}

Two reviewers will independently extract and manage the data for each of the included studies using an electronic data extraction form. We will pilot the data extraction form and amend it according to feedback received from a panel of experienced colleagues. We plan to contact study authors in case of any unclear or missing information. Disagreements between review authors will be resolved by discussion. A third review author will act as an arbiter in case disagreements cannot be resolved. Variations that may depend on agedistribution, the categorisation of ulcer, study design etc. will be described clearly.

\section{Data items}

Data will be extracted on the following.

1. Publication details: title, journal, author, year, city and country, in which the study was conducted, type of publication, and source of funding.

2. Design: type of study (observational studies, crosssectional, cohort, case-control, single arm studies, systemic review/meta-analyses); aims of study, method of data collection, response rate, recruitment and sampling methods, eligibility (inclusion and exclusion criteria).

3. Study participant details: number of persons interviewed or surveyed, population characteristics including setting, age, gender, ethnicity, demographic information, diagnostics, ulcer specifications (including stage), complications.

4. Data for outcome measures: all reported estimates, or sufficient information to calculate an estimate of the point prevalence, period prevalence, cumulative incidence and incidence rate of chronic wounds in the categories of pressure ulcers, diabetic ulcers, venous ulcers and arterial ulcers, and complications thereof in four categories (infections, gangrene, haemorrhage and limb amputation).

5. Limitations: selection bias, response bias, information bias, limitations of assessment tool(s) used and limitations reported by study authors.

\section{Risk of bias in individual studies}

The risk of bias in included studies will be assessed using a tool developed specifically for conducting quality appraisal of studies in systematic reviews of prevalence data [18]. The instrument addresses critical issues of internal and external validity that must be considered when assessing validity of prevalence data and can be used across different study designs. The instrumental framework evaluates representativeness, recruitment, sample size, reporting, data coverage, condition reliability, statistical analysis and confounding factors using a simple "yes", "no", "unclear" or "not/applicable". The outcome of the assessment will be presented in a table.

Publication bias and selective reporting will be dealt with by critically assessing study findings, plots will be made of outcome variables against sample size [19] and advice will be taken from GRADE guidelines No 5 [20]. 


\section{Data synthesis}

Relevant data extracted from eligible studies will be presented in evidence tables. Meta-analysis of outcome variables will be conducted if studies are adequately meeting the inclusion criterion and uniform (or clarity) in reporting the outcome estimates. A narrative synthesis will provide summary of the prevalence of chronic wounds and complications thereof according to age, setting and category of wound. These grouping variables will also be used to identify similar patient populations so that quantitative meta-analyses could be made for studies with similar design. The extracted incidence and prevalence figures will be presented with corresponding standard error and $95 \%$ confidence intervals using the exact binomial method as described by Clopper and Pearson [21]. This method produces an exact confidence interval that is directly based on the binomial distribution rather than any approximation to the binomial distribution.

Further, the individual prevalence and incidence rate estimates from observational studies will be pooled using the fixed effect and random effect models [22]. The choice of random-effects model will be based on clinical and methodological diversity across the included studies. We will also perform cumulative meta-analysis, in which estimates for each year will be determined in order to calculate the impact of each added study on the pooled estimates over time. For country-specific pooling of incidence/prevalence data, the Poisson regression technique will be used to adjust the covariates [23]. Where available, the prevalence-adjusted or incidence-adjusted odds ratios relating to the stratification of risk of chronic wound among the patients as discussed in the respective observational studies will also be pooled using random effect models. We will investigate the potential source of heterogeneity related to both methodological and clinical characteristics of the studies which will be assessed by the Cochran's $Q$ test ( $P$ value $<0.05$ considered significant) and $I^{2}$ (>50\% representing moderate heterogeneity) statistics [24]. In the presence of heterogeneity $\left(I^{2}>25 \%\right)$ [25], subgroup (e.g. age, sex, setting and clinical categories of chronic wounds) analyses and univariate meta-regression $(P$ value $<0.10$ considered significant given the low power of these tests) will be carried out in order to estimate the effect of study-level covariates on the estimates of incidence and prevalence [26]. Sensitivity analyses will be conducted to identify the study-level factors that best describe the occurrence of chronic wounds. We will also perform influence and outlier analyses to determine the effects of certain studies on the pooled estimates of chronic wound occurrence [27]. These analyses will measure how the estimated parameter of a pooledanalysis would change if noisy studies are eliminated.
The effect of these identified outlier studies will be neutralised by excluding them from the random effect model. The publication bias due to study size will also be addressed and adjusted by inverse-variance weighting techniques to provide valid information on the study estimates [26].

\section{Summary}

This systematic review and eventually meta-analysis will be performed to critically examine the world's relevant literature on the epidemiology of chronic wounds. Specifically, we aim to identify and report the estimated prevalence and incidence of chronic wounds in the categories of pressure ulcers, diabetic ulcers, venous ulcers and arterial insufficiency ulcers and related complications for different settings and subgroups. We will also identify population characteristics associated with chronic wounds in the four categories and with the following complications: infections, gangrene, haemorrhage and limb amputation. Understanding the rates of different categories of complications among patients with chronic wounds could help target patient subgroups that may benefit from early screening, prevention, and treatment efforts. In addition, quantifying the magnitude of chronic wounds will help guide decision-making for the allocation of scarce healthcare resources and funding. A further finding of this systematic review will be the result of the methodological assessment of the published literature. The findings of this review will also be compared with other similar published reviews. Finally, conclusions will be drawn from this systematic review highlighting the prevalence and incidence of chronic wounds, methods of estimation and settings and their correlates. Limitations of the studies will be discussed in detail. Implications of the review as well as suggestions for future research will also be provided.

\section{Additional files}

Additional file 1: PRISMA-P checklist. (DOC $84 \mathrm{~kb}$ )

Additional file 2: MEDLINE (Ovid) search strategy. (DOCX $32 \mathrm{~kb}$ )

\section{Acknowledgements \\ We are grateful to Carl Gornitzki at the university library at the Karolinska Institutet, Sweden, for invaluable support in the refinement of the search strategies.}

\section{Funding}

This research is supported by the A*STAR BMRC Strategic Positioning Fund (SPF) for Skin Biology awarded to the Institute of Medical Biology, and through internal grant funding from the Lee Kong Chian School of Medicine, Nanyang Technological University. The views expressed are those of the authors and not necessarily those of the NTU or A*STAR. Lee Kong Chian School of Medicine, NTU and A*STAR have no role as organisations in the planning, conduct or dissemination of the systematic review. 


\section{Authors' contributions}

$\mathrm{KJ}$ and GN initiated the protocol, conceptualised the research plan for the proposed systematic review, wrote the manuscript and reviewed it for important intellectual content. HS, AS, CP, RB and JC critically reviewed the methodology, wrote the manuscript and reviewed it for important intellectual content. All authors read and approved the final manuscript.

\section{Competing interest}

The authors declare that they have no competing interests.

\section{Consent for publication}

Not applicable.

\section{Ethics approval and consent to participate} Not applicable.

\section{Author details}

'Centre for Population Health Sciences (CePHaS), Lee Kong Chian School of Medicine, Nanyang Technological University, 59 Nanyang Drive, Experimental Medicine Building, Singapore 636921, Singapore. ${ }^{2}$ Department of Dermatology, Skåne University Hospital, Lasarettsgatan 15, 22185 Lund, Sweden. ${ }^{3}$ Dermatology and Innate Immunity Laboratory, Lee Kong Chian School of Medicine, Nanyang Technological University, 59 Nanyang Drive, Experimental Medicine Building, Singapore 636921, Singapore. ${ }^{4}$ Medical Library, Lee Kong Chian School of Medicine, 11 Mandalay Road, Singapore 636921, Singapore. ${ }^{5} \mathrm{Global}$ eHealth Unit, Department of Primary Care and Public Health, School of Public Health, Imperial College London, 3rd Floor Reynolds Building, St Dunstan's Road, London W6 8RP, UK

Received: 29 April 2016 Accepted: 31 August 2016

Published online: 08 September 2016

\section{References}

1. Lazarus GS, Cooper DM, Knighton DR, Percoraro RE, Rodeheaver G, Robson MC. Definitions and guidelines for assessment of wounds and evaluation of healing. Wound Repair Regen. 1994:2:165-70.

2. Werdin F, Tennenhaus M, Schaller HE, Rennekampff HO. Evidencebased management strategies for treatment of chronic wounds. Eplasty. 2009;9, e19.

3. Mekkes JR, Loots MAM, Van Der Wal AC, Bos JD. Causes, investigation and treatment of leg ulceration. Br J Dermatol. 2003. doi:10.1046/j.1365-2133. 2003.05222.

4. Cazander G, Pritchard DI, Nigam Y, Jung W, Nibbering PH. Multiple actions of Lucilia sericata larvae in hard-to-heal wounds. Bioessays. 2013. doi:10. 1002/bies.201300071.

5. The Wound Healing Society. Chronic wound care guidelines. 2006. http://woundheal.org/documents/final_pocket_guide treatment.aspx. Accessed 20 Nov 2015

6. Sen CK, Gordillo GM, Roy S, Kirsner R, Lambert L, Hunt TK, et al. Human skin wounds: a major and snowballing threat to public health and the economy. Wound Repair Regen. 2009. doi:10.1111/j.1524-475X.2009.00543.X.

7. Gottrup F. A specialized wound-healing center concept: importance of a multidisciplinary department structure and surgical treatment facilities in the treatment of chronic wounds. Am J Surg. 2004;187(5):S38-43.

8. Wicke C, Bachinger A, Coerper S, Beckert S, Witte MB, Königsrainer A. Aging influences wound healing in patients with chronic lower extremity wounds treated in a specialized Wound Care Center. Wound Repair Regen. 2009 doi:10.1111/j.1524-475X.2008.00438.x.

9. Kloth $\mathrm{L}$. The roles of physical therapists in wound management, part II: patient and wound evaluation. J Am Col Certif Wound Spec. 2009. doi:10. 1016/j.jcws.2009.03.003.eCollection2009.

10. Margolis D. Epidemiology of wounds. In: Romanelli M, Shukla V, Mani R, editors. Measurements in Wound Healing. London: Springer; 2013. p. 145-53.

11. MacDonald J. Global initiative for wound and lymphoedema care (GIWLC). Journal of Lymphoedema. 2009;4(2):92-5.

12. Heyer K, Augustin M, Protz K, Herberger K, Spehr C, Rustenbach S Effectiveness of advanced versus conventional wound dressings on healing of chronic wounds: systematic review and meta-analysis. Dermatology. 2013. doi:10.1159/000348331. Epub 2013 May 22.
13. Denny K, Lawand C, Perry S. Compromised wounds in Canada. Healthcare Quarterly (Toronto, Ont). 2013;17(1):7-10.

14. Posnett J, Franks PJ. The burden of chronic wounds in the UK. Nurs Times. 2008;104(3):44-5.

15. Graves N, Zheng $H$. The prevalence and incidence of chronic wounds: a literature review. Wound Practice and Research. 2014;22(1):4-19.

16. Moher D, Shamseer L, Clarke M, Ghersi D, Liberati A, Petticrew M, Shekelle P, Stewart LA, PRISMA-P Group. Preferred reporting items for systematic review and meta-analysis protocols (PRISMA-P) 2015 statement. Syst Rev. 2015. doi:10.1186/2046-4053-4-1.

17. The National Pressure Ulcer Advisory Panel - NPUAP. National Pressure Ulcer Advisory Panel (NPUAP) announces a change in terminology from pressure ulcer to pressure injury and updates the stages of pressure injury. Washington DC April 13 2016. http://www.npuap.org/national-pressureulcer-advisory-panel-npuap-anno. Assessed 15 Aug 2016

18. Munn Z, Moola S, Riitano D, Lisy K. The development of a critical appraisal tool for use in systematic reviews addressing questions of prevalence. Int J Health Policy Manag. 2014;3(3):123-8.

19. Egger M, Smith GD, Schneider M, Minder C. Bias in meta-analysis detected by a simple, graphical test. BMJ. 1997;315:629-34.

20. Guyatt GH, Oxman AD, Montori V, Vist G, Kunz R, Brozek J, Alonso-Coello P, Djulbegovic B, Atkins D, Falck-Ytter Y, Williams Jr JW, Meerpohl J, Norris SL, Akl EA, Schunemann HJ. GRADE guidelines: 5. Rating the quality of evidence_-publication bias. J Clin Epidemiol. 2011:64:1277-82.

21. Clopper CJ, Pearson ES. The use of confidence or fiducial limits illustrated in the case of the binomial. Biometrika. 1934;26:404-13.

22. DerSimonian R, Laird N. Meta-analysis in clinical trials. Control Clin Trials. 1986;7(3):177-88.

23. Chan KY, Wang W, Wu JJ, Liu L, Theodoratou E, Car J, Middleton L, Russ TC, Deary IJ, Campbell H, Rudan I. Epidemiology of Alzheimer's disease and other forms of dementia in China, 1990-2010: a systematic review and analysis. Lancet. 2013. doi:10.1016/S0140-6736(13)60221-4.

24. Scott BN, Roberts DJ, Robertson HL, Kramer AH, Laupland KB, Ousman SS, Kubes $P$, Zygun DA. Incidence, prevalence, and occurrence rate of infection among adults hospitalized after traumatic brain injury: study protocol for a systematic review and meta-analysis. Syst Rev. 2013. doi:10.1186/2046-4053-2-68.

25. Higgins JPT, Thompson SG, Deeks JJ, Altman DG. Measuring inconsistency in meta-analyses. BMJ. 2003;327:557-60.

26. Borenstein M, Hedges LV, Higgins JPT, Rothstein HR. Front matter, in introduction to meta-analysis. Chichester, UK: John Wiley \& Sons Ltd; 2009. doi:10.1002/9780470743386.

27. Higgins JP. Green S. Cochrane handbook for systematic reviews of interventions version 5.1. 0. The Cochrane Collaboration, 2011. http:// handbook.cochrane.org/. Assessed 28 Mar 2016.

\section{Submit your next manuscript to BioMed Central and we will help you at every step:}

- We accept pre-submission inquiries

- Our selector tool helps you to find the most relevant journal

- We provide round the clock customer support

- Convenient online submission

- Thorough peer review

- Inclusion in PubMed and all major indexing services

- Maximum visibility for your research

Submit your manuscript at www.biomedcentral.com/submit
Biomed Central 